\title{
ANÁLISIS DE LAS RELACIONES DE PREFERENCIA ENTRE FRUTOS COMPONENTES DE LA DIETA DE LA CORZUELA PARDA Mazama gouazoubira Fischer (MAMMALIA, CERVIDAE), EN UN AMBIENTE SECUNDARIO DE YUNGAS (TUCUMÁN - ARGENTINA)
}

\section{FRUIT PREFERENCE ANALYSIS IN THE DIET OF THE BROWN BROCKET DEER Mazama gouazoubira Fischer (MAMMALIA, CERVIDAE), IN A SECONDARY YUNGAS ENVIRONMENT (TUCUMÁN - ARGENTINA)}

\author{
Enrique Richard ${ }^{1,2}$ y Francisco Fontúrbel Rada ${ }^{3 *}$
}

\begin{abstract}
Resumen
Se analizó la preferencia de frutos de la dieta de Mazama gouazoubira en un ambiente de bosque secundario de Yungas (Tucumán, Argentina). El consumo de frutos de Morus nigra L. representa una porción muy importante de la dieta de $M$. gouazoubira bajo las condiciones del ambiente del área de estudio (un ambiente secundario de la Provincia Fitogeográfica de Las Yungas); otros frutos consumidos por M. gouazoubira son Psychotria carthagenensis Jacq. y en mucha menor proporción, Rubus boliviensis Focke. Se analizaron las preferencias alimentarias ofreciendo el mismo fruto a diferentes densidades relativas en condiciones de semicautiverio, donde se evidenció de la preferencia $M$. nigra, incluso ante una abundancia elevada de $P$. Carthagenensis y $R$. boliviensis. Este último fue, más bien, el fruto más rechazado por M. gouazoubira. Este comportamiento de selección independiente de la densidad se puede explicar por tres razones: (1) La relación pulpa / semilla y la composición fotoquímica de M. nigra son óptimas para la dieta de la corzuela parda, (2) M. nigra es más palatable que $P$. carthagenensis, y (3) El alto contenido de fibra de $R$. boliviensis que hace a sus frutos difíciles de digerir, y que por ende, son frecuentemente rechazados.
\end{abstract}

Palabras clave: Preferencia, dieta, ambiente secundario, Yungas, Mazama gouazoubira, palatabilidad

\begin{abstract}
Morus nigra L. fruit consumption by the brown brocket deer, Mazama gouazoubira, represents a very significant part of its diet in the area under study (a secondary environment of the Phitogeografical Province of Las Yungas). Other important fruits in the area are Psychotria carthagenensis Jacq. and, to a lesser extent, Rubus boliviensis Focke. Fruit preferences were analyzed, offering the same fruits, at different relative densities, to brocket deers at semicaptivity conditions. They always preferred $M$. nigra, even at very high $P$. carthagenensis densities, while $R$. boliviensis was the most rejected. The best relation pulp/seed and the highest nutritional value of $M$. nigra would be the reasons for its high palatability compared to $P$. carthagenensis. In spite of having intermediate values compared to the other fruits considered, the high fiber content and the subsequent low digestibility of $R$. boliviensis, would be the reasons for its rejection.
\end{abstract}

Keywords: Preference, diet, secondary environment, Yungas, Mazama gouazoubira, palatability

\section{Introducción}

Los ungulados neotropicales juegan un importante rol en la determinación de la composición específica y estructural de los bosques en que habitan, a través de la depredación y/o dispersión de semillas de frutos comestibles (Bodmer, 1989; Stallings, 1984; Terborgh, 1992; Varela \& Brown, 1995). La determinación de la preferencia de frutos que consumen en la dieta de tales animales constituye un primer paso hacia la comprensión y/o predicción de los cambios estructurales en la comunidad que estos pueden ocasionar, especialmente en ambientes secundarios. La selva pedemontana (Prado, 1995) como parte de la Provincia Fitogeográfica de Las Yungas (Cabrera, 1971), es un ecosistema prácticamente extinto en la provincia de Tucumán y cuyos remanentes se encuentran en un $100 \%$ afectados en algún grado por la actividad humana (Vides et al., 1998), constituyendo por lo tanto ambientes secundarios de esta ecoregión. Dentro de este ambiente, el Moradillo (Psychotria carthagenensis Jacq., Rubiaceae) ha sido propuesto como una especie clave para las comunidades de frugívoros, dado que fructifica en gran cantidad y en una época en que escasean frutos comestibles (Vides et 
al., 1993; Arias \& Parrado, 1996; Capllonch et al., 2001).

Entre los ungulados que habitan la selva pedemontana, la corzuela parda (Mazama gouazoubira Fischer, Mammalia: Cervidae), es uno de los que posee una las dietas más marcadamente frugívora (Bodmer, 1989, 1991; Juliá et al., 1993; Richard et al., 1995a b), aunque la importancia de este ítem trófico está directamente vinculada a su disponibilidad fenológica (Richard et al., 1995a). Asimismo, la corzuela colorada, M. americana (Mammalia: Cervidae), posee de igual modo, una dieta altamente frugívora (Branan et al., 1985; Bodmer, 1989, 1991), pero ésta parece habitar en zonas de mayor altura y sería muy rara o ausente en la ecoregión de Selvas Pedemontanas (Richard et al., 1995 b).

La dieta de la M. gouazoubira ha sido registrada exhaustivamente en la Reserva Experimental Horco Molle (REHM, provincia de Tucumán, Argentina) en donde, entre los meses de septiembre a noviembre el consumo de moras (Morus nigra L., Moraceae), constituye la casi totalidad de frutos presentes en la misma (Juliá et al., 1993; Richard et al., 1995a b). Sin embargo, durante este período otros frutos se encuentran también disponibles en el área, como el moradillo y la zarza mora (Rubus boliviensis Focke, Rosaceae), a pesar de lo cual su consumo no ha sido registrado dentro de la dieta en estudios de campo previos (Juliá et al., 1993; Richard et al., 1995a b).

La importancia de la mora dentro de la dieta de la corzuela parda en la REHM podría deberse a la mayor abundancia de esta exótica dentro del área de estudio en relación con las otras especies consideradas ( $P$. carthagenensis y $R$. boliviensis). Estas últimas, podrían constituir fuentes alternativas de fruta, en áreas donde la mora está ausente o su frecuencia en la dieta podría aumentar, conforme aumenta su disponibilidad. Por su mayor abundancia en la zona y accesibilidad para las corzuelas (dada la menor altura a la que se encuentran los frutos), el moradillo sería potencialmente mucho más importante que la zarza mora como fruto alternativo en la dieta de este cérvido. Sin embargo, dado que la ausencia de estas especies en la dieta pudiera deberse a otro factor además de su relativa baja disponibilidad en el área; determinar su palatabilidad para la corzuela y el grado de preferencia que esta exhibe frente a los distintos frutos presentes en la zona, podría dar una idea sobre su importancia relativa para estos frugívoros - folívoros de mediano porte.

Es importante destacar que el fruto más consumido por la corzuela, en el mes más crítico en cuanto a la oferta de este recurso (septiembre) en esta parte de las Yungas (Capllonch et al., 2001), es la mora, fruto que para la región es exótico.

Luego, los objetivos del presente trabajo están dirigidos a dar respuesta a los siguientes interrogantes surgidos del análisis precedente:
¿Es el moradillo un fruto palatable para $M$. gouazoubira?

¿Cuál es el orden de preferencia entre los frutos considerados?

¿La densidad del moradillo influye en la selección de moras?

¿Qué relación existe entre digestibilidad y valor nutricional de los frutos de mora, moradillo y zarza mora, con el grado de preferencia de la corzuela por los mismos?

\section{Materiales y métodos}

Área de estudio

El área de estudio está incluida dentro de la Reserva Experimental de Flora y Fauna de Horco Molle (REHM) dependiente de la Facultad de Ciencias Naturales e Instituto Miguel Lillo, Universidad Nacional de Tucumán. Esta consta de 200 ha. Un grupo de corzuelas (19) bajo manejo se ubican en un cercado perimetral de 18 ha en donde se encuentran los individuos objeto de estudio. Mazama gouazoubira es un cérvido que además se encuentra en forma relativamente abundante fuera del cercado, en la REHM y región perimetral a la misma. La REHM se ubica al pie oriental de las sierras de San Javier (entre $26^{\circ} 38^{\prime}$ y $26^{\circ} 57^{\prime}$ lat. sur y $65^{\circ} 26^{\prime}$ y $65^{\circ} 20^{\prime}$ long. oeste), en Horco Molle, Dpto. Yerba Buena, Tucumán, Argentina. La REHM está incluída, en toda su extensión, dentro de lo que actualmente se conoce como la ecoregión de la Selvas Pedemontanas (Vides Almonacid et al., 1998). Dicha unidad, se engloba dentro del Distrito de la Selva Montana de la Provincia Fitogeográfica de las Yungas, según Cabrera (1971). Sin embargo, más recientemente (Prado, 1995) la ecoregión de Selvas Pedemontanas ha sido catalogada como una unidad diferente de las Yungas, en función de sus particularidades biológicas y su alto grado de intervención, que a los fines de su tratamiento, conservación y manejo justificarían tal decisión. Sin embargo y dado que la totalidad de trabajos previos en el tema (Juliá, 1993; Richard et al., 1995a Richard et al., 1995 b) refieren a los ecosistemas de la REHM como parte de la Provincia Fitogeográfica de las Yungas es que, a los fines prácticos, se mantendrá dicha asignación. 


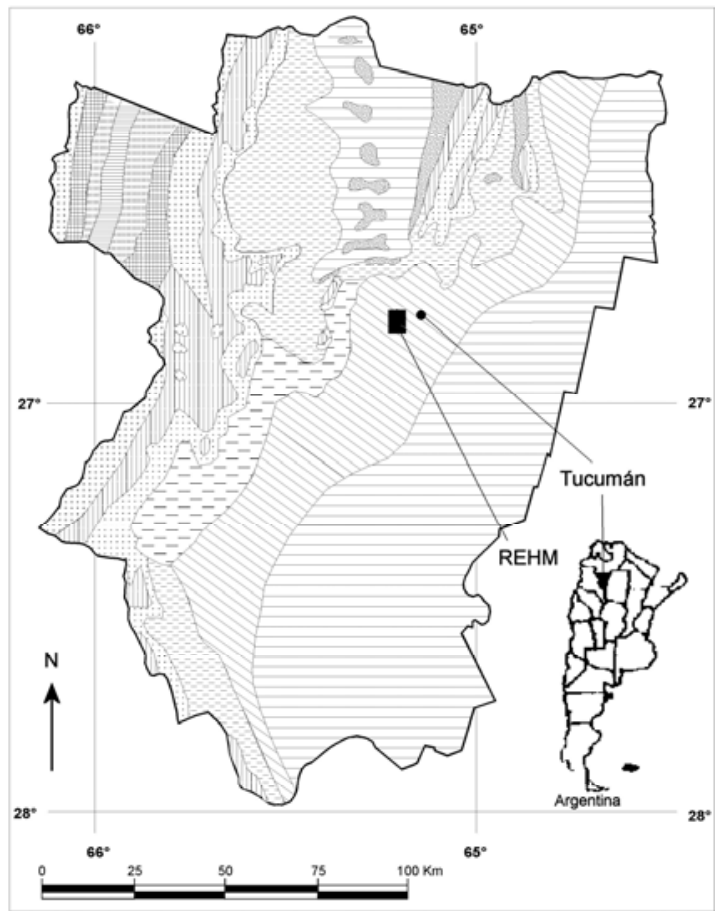

Figura 1. Ubicación geográfica y biogeográfica relativa del área de estudios (REHM). Modificado de Cabrera (1971), Prado et al. (1995) y Versvoorst (1982).

\section{Diseño experimental}

El período de estudio abarcó los meses de Septiembre y Octubre de 1994, período durante el cual existe gran disponibilidad de los frutos considerados en el área en cuestión y época considerada crítica en cuanto a la oferta de frutos (Capllonch et al., 2001). En el área se observan distintos ambientes secundarios de la selva pedemontana y un gran número de especies exóticas establecidas en tiempo relativamente reciente (últimos 50 años). Las especies vegetales consideradas, $M$. nigra, $P$. carthagenensis, principalmente, y $R$. boliviensis, son abundantes en la zona y de ellas sólo las dos últimas son autóctonas. cuales una muestra fue utilizada para llevar a cabo un análisis proximal de Weende (Tabla 1).

Para responder a los interrogantes planteados como objetivos del presente trabajo, se idearon las siguientes experiencias:

Palatabilidad del moradillo: A fin de comprobar la validez del moradillo como fruto alternativo (ya que su consumo sólo se presumía en el área), fue ofrecida a todas los ejemplares de corzuela con el objeto de verificar su aceptación. Como ejemplar testigo se utilizó un macho aislado, que nunca tuvo contacto previo con frutos de mora. A este se le suministraron frutos de mora y moradillo.

La preferencia entre frutos se probó colocando en el centro de un paño mimético de $50 \mathrm{~cm}$ de lado, un arbusto armado con ramas de moradillo con entre 300 a 500 frutos $y$, alrededor del mismo, y en forma aleatoria, 50 frutos de mora. Los frutos así colocados, se encontraban de la misma forma en que están disponibles en forma natural para las corzuelas, aunque en densidades mucho mayores. Los animales (dos machos y una hembra en este caso), comían en distintos turnos durante 20 segundos; luego de los cuales se registraba la cantidad de frutos consumidos de cada especie vegetal y se reponían los faltantes. También se ofrecieron frutos de zarza mora a fin de determinar su potencial atractivo para las corzuelas, por considerarlo una tercera alternativa disponible para las mismas durante el período de estudio.

El efecto de la densidad de frutos de moradillo sobre el consumo de moras se evaluó colocando ambos frutos a densidades relativas mucho mayores para el primero (1408-8400 frutos $/ \mathrm{m}^{2}$ frente a $140-200$ frutos $/ \mathrm{m}^{2}$ ) y registrando el consumo cada 20 segundos sin reposición.

A los fines de utilizar unidades comparables de consumo de frutos, se pesó 10 veces, 10 frutos de mora y zarza mora y 100 frutos de moradillo, con una balanza marca Pesola de 300 gramos; en cada repetición se dividió el peso obtenido por el número de frutos, para obtener la biomasa promedio de cada fruto.

Tabla 1: Resultados del análisis proximal de Weende para los frutos considerados.

\begin{tabular}{lllllllll}
\hline Especie & $\begin{array}{l}\text { Humedad } \\
\text { \% }\end{array}$ & $\begin{array}{l}\text { Materia } \\
\text { seca \% }\end{array}$ & $\begin{array}{l}\text { Proteína } \\
\text { bruta \% }\end{array}$ & $\begin{array}{l}\text { Extracto } \\
\text { etéreo \% }\end{array}$ & $\begin{array}{l}\text { Fibra } \\
\text { cruda \% }\end{array}$ & $\begin{array}{l}\text { Ceniza } \\
\text { \% }\end{array}$ & $\begin{array}{l}\text { Hidratos } \\
\text { carbono \% }\end{array}$ & $\begin{array}{l}\text { Relación } \\
\text { nutrit.: 1 }\end{array}$ \\
\hline $\begin{array}{l}\text { Morus nigra } \\
\begin{array}{l}\text { Rubus } \\
\text { boliviensis }\end{array}\end{array}$ & 80.13 & 19.87 & 10.50 & 9.01 & 6.25 & 5.65 & 68.59 & 9.19 \\
$\begin{array}{l}\text { Psychotria } \\
\text { carthagenensis }\end{array}$ & 82.04 & 17.96 & 9.62 & 8.25 & 23.12 & 4.00 & 55.01 & 10.18 \\
& 80.24 & 19.76 & 7.44 & 5.52 & 17.02 & 3.65 & 66.37 & 12.99
\end{tabular}

A fin de determinar el grado de preferencia por uno u otro fruto, se realizaron test de "cafetería" (sensu Braza et al., 1994 a) con cuatro ejemplares adultos de corzuela parda (tres machos y una hembra), en condiciones de semicautiverio dentro del cercado. Para ello se colectaron frutos en el área de estudio, de los
En las pruebas realizadas se contaron el número de frutos utilizados y los posteriormente consumidos y se multiplicaron por la biomasa promedio de cada uno de ellos.

Para correlacionar el valor nutritivo de los frutos con el grado de preferencia de las corzuelas por ellos 
se utilizaron los datos del análisis proximal de Weende.

\section{Resultados y Discusión \\ Palatabilidad del Moradillo}

Los individuos de $M$. gouazoubira consumieron frutos de moradillo siempre que no hubiera moras presentes. El ejemplar aislado (un macho de un año y medio), prefirió primero únicamente frutos de moradillo $(n=3)$, pese a que se le suministraron ambos tipos de frutos durante tres días consecutivos. Este animal nunca tuvo contacto previo con frutos de mora. Los frutos de mora en este caso, eran consumidos únicamente cuando se agotaba el primer fruto, las hojas de mora (las cuales había tenido oportunidad de comer con anterioridad) fueron seleccionadas en primer término y en mayor cantidad que los frutos de esta especie. Sin embargo, luego de liberar al animal en el cercado (teniendo gran cantidad de frutos de moras a su disposición) y tras una semana, cambió su preferencia eligiendo moras, aún a densidades altas de frutos de moradillo. Esto confirma la idea de que los frutos de moradillo son también atractivos para las corzuelas, al menos en ausencia de moras. La ausencia de registros de consumo de moradillo previo a los test de cafetería se debería a su escasez dentro del cercado (no así en el resto de la REHM).

El moradillo fue ofrecido en forma de frutos sueltos en un paño y en racimos (por encontrarse de ambas formas disponible naturalmente para las corzuelas). Estas ignoran por lo general los frutos de moradillo dispersos en el suelo y consumen solo aquellos presentes en estado agrupado (en racimos) (Figura 1), por lo que en las pruebas estos fueron dispuestos en esa forma.

Consumo de frutos sueltos y agrupados (racimo) de Moradillo

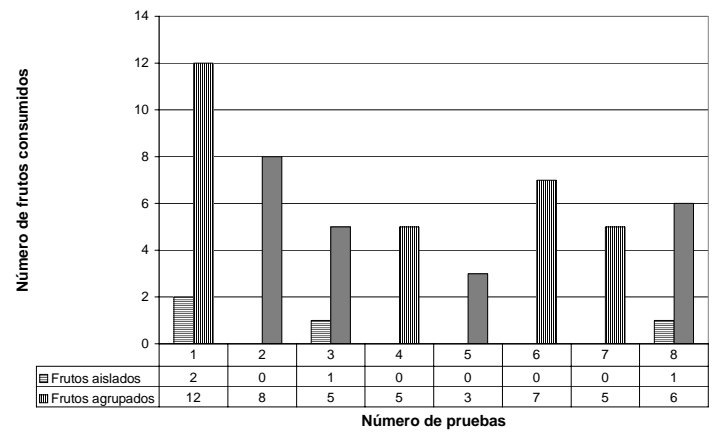

Figura 2. Consumo de frutos de moradillo (Psychotria carthagenensis) sueltos y agrupados en racimos. Para esta prueba se utilizaron dos machos adultos.

Dado que los frutos de zarza mora fueron generalmente rechazados y dejados de lado por los otros frutos, no se utilizaron en los test. Sólo en dos oportunidades de 15 intentos, en días distintos y con diferentes animales, se registró su consumo (tres y siete frutos), en la primera oportunidad no había frutos alternativos.

Preferencia

Al comparar la preferencia por uno u otro fruto, los frutos de mora fueron consumidos en forma significativamente superior $(\mathrm{P}<0.01$; test de $\mathrm{U}$ - Mann - Withney, Figura 2). En este caso los frutos de moradillo fueron generalmente ignorados.

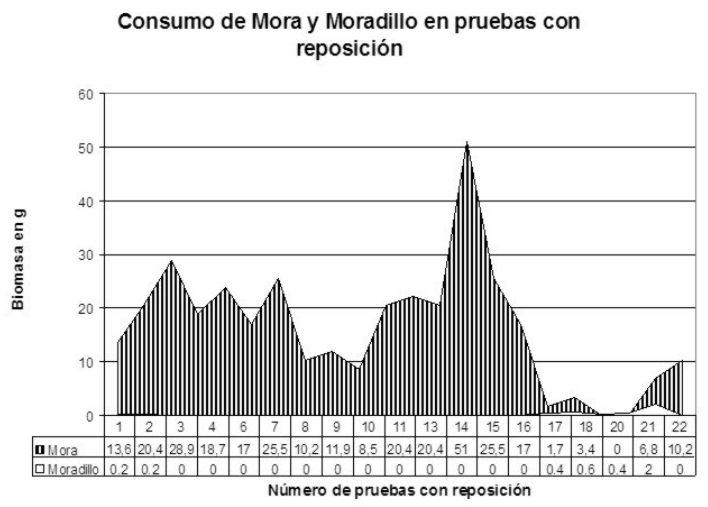

Figura 3. Consumo de mora (M. nigra) y moradillo ( $P$. carthagenensis) $(n=22)$, en pruebas con reposición. Se observaron diferencias altamente significativas en el consumo de uno y otro fruto. U-Mann-Whitney; $\mathrm{P}=$ 0.01. Consumo medio de $M$. nigra $=16.23 \mathrm{~g}$; consumo medio de $P$. carthagenensis $=0.18 \mathrm{~g}$

\section{El efecto de la densidad}

Ante densidades relativas bajas de frutos de mora (140 - 200/m² o 59.5 g y 81.6 g), entremezcladas con una muy alta densidad de frutos de moradillo (1408 $8400 / \mathrm{m}^{2}$ o 70.4 g y 420 g), las corzuelas seleccionaron cuidadosamente las moras hasta casi agotar este recurso. Sólo a biomasas de moradillo entre 2 y 10 veces superiores a las de mora el consumo de moradillo aumentaba levemente (Figuras 3 y 4).

Valor nutritivo y selección

Las diferencias en contenido de hidratos de carbono entre los frutos de moradillo y mora no parecen ser biológicamente significativas. La elección entre ellos por lo tanto, no sería debido a esta causa. La mora posee relativamente más proteína bruta, factor que puede convertirse en limitante (Braza et al., 1994b); asimismo posee más grasas (extracto etéreo), menos fibra y una mejor relación nutritiva (Tabla 1). También posee el mayor porcentaje de cenizas, el cual estaría relacionado positivamente con la palatabilidad (Nagy \& Haufler, 1987). Estudios realizados con aves frugívoras (Wilson \& Whelan, 1993) demuestran que los frutos de Cornus drummondii (Cornaceae) con altos contenidos de grasas resultan más atractivos para algunas de ellas, por lo que tal situación podría influir también en la selección de la corzuela. Finalmente, la preferencia por la mora podría deberse a que ésta se presenta como un recurso más conspicuo en cuanto a 
relación pulpa - semilla y tamaño relativo, el cual también es un factor importante de preferencia para muchas aves (Wilson \& Whelan, 1993). Sin embargo, la importancia de las semillas como fuente de alimento para estos cérvidos (Bodmer, 1989), debería inclinar la preferencia hacia el lado del moradillo.

Consumo de frutos de Mora y Moradillo a distintas biomasas (biomasa inicial de Moradillo 1,2 veces superior)

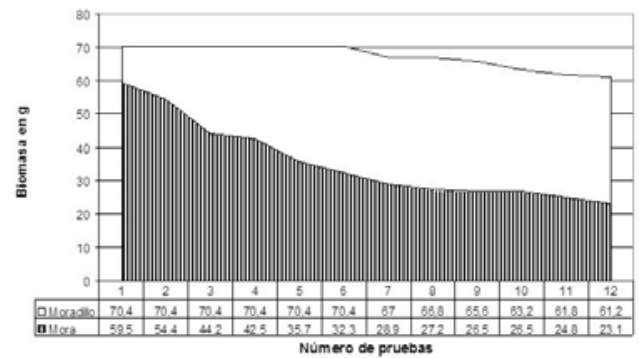

Figura 4: Curvas de supervivencia de frutos de mora (M. nigra) y moradillo ( $P$. carthagenensis) en once muestras sin reposición; $\mathrm{n}^{\circ}$ inicial de moras $=35$, número inicial de $P$. carthagenensis $=352$.

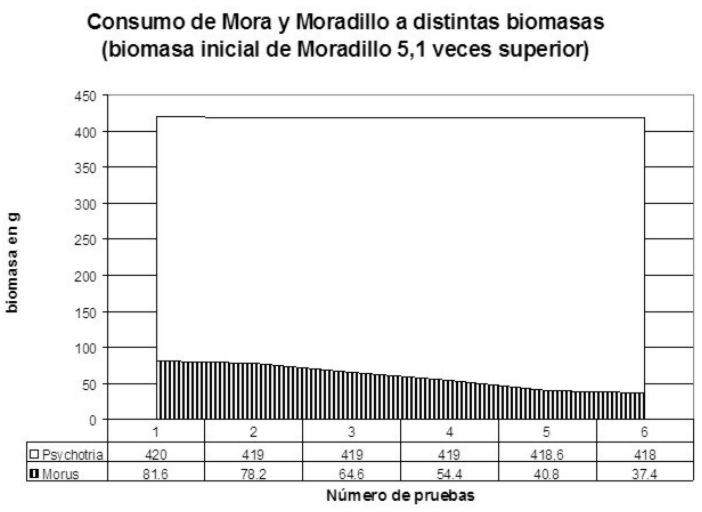

Figura 5: Curvas de supervivencia de frutos de mora (M. nigra) y moradillo ( $P$. carthagenensis) en seis muestras sin reposición; $\mathrm{n}^{\circ}$ inicial de moras $=48$, número inicial de $P$. carthagenensis $=2100$.

Otro factor que debe ser considerado, probablemente el más importante, está referido a las propiedades raticidas del género Psychotria y la presencia de idioblastos de oxalato cálcico en sus frutos. Dicho compuesto químico, en grandes concentraciones, es tóxico para mamíferos y su efecto en la corzuela se desconoce actualmente. Los frutos de zarza mora poseen valores intermedios en cuanto a porcentajes de proteína bruta, grasas y relación nutritiva, aunque posiblemente el bajo nivel de carbohidratos y especialmente, el alto nivel de fibras (que disminuyen su digestibilidad) se constituyan en la causa más probable de su baja palatabilidad (Tabla 1).
La corzuela, al igual que otros rumiantes pequeños (Gwynne \& Bell, 1968; Bell, 1971; Hobbs et al., 1983), posee una relativamente baja capacidad para digerir alimentos fibrosos (Putman, 1988), por lo que este se constituye en factor determinante en el grado de selección de los componentes de la dieta.

Según Pulliam (1974) la estrategia especialista es independiente de la densidad de presas alternativas. En el caso de la corzuela tal densidad (moradillo), fue llevada a niveles muy superiores de disponibilidad respecto a mora, sin que se modifique la preferencia por esta última.

De lo expuesto, la corzuela parda se comporta como un especialista en el sentido de Pulliam (1974), que preferiría la mora sobre el moradillo sin importar la densidad de este último. En el período de estudio, meses de septiembre y octubre, la mora representa aproximadamente entre el $20 \%$ y el $40 \%$ de la dieta (Richard et al., 1995 a) de todos los ejemplares de corzuela del cercado (19 individuos).

La digestibilidad y valor nutritivo de la mora, sumados a su abundancia (Richard et al., 1995 a) justifican una disminución en la diversidad potencial de la dieta, dejando de lado el consumo de otras partes vegetales. Esto último concuerda con lo propuesto por Nudds (1980) al teorizar sobre las razones que determinarían las preferencias de forrajeo del ciervo de cola blanca (Odocoileus virginianus). La estrategia trófica observada en este trabajo se encuentra también, en concordancia con la teoría de forrajeo óptimo de MacArthur \& Pianka (1968), ya que permite a la corzuela maximizar su tasa de ingesta de nutrientes por unidad de tiempo, priorizando el valor nutricional y la digestibilidad del alimento. De esta manera, estos cérvidos (Mazama spp.) cumplen con los dos objetivos de la búsqueda de alimento de todo herbívoro (Braza et al., 1994 b): conseguir el mayor número de nutrientes y reducir el tiempo dedicado a la alimentación.

La importancia que pueden tener las corzuelas en la regeneración de la selva pedemontana, no ha sido evaluada, pero para el área de estudio considerada, es importante determinar su rol como dispersor y/o depredador de semillas de especies exóticas como la mora.

\section{Conclusiones}

Los resultados obtenidos por el test de "cafetería" sugieren que el moradillo ( $P$. carthagenensis) es poco palatable para las corzuelas, y por ende sus frutos no son altamente consumidos, aún en condiciones de abundancia.

El análisis de las preferencias alimenticias de las corzuelas ofreciendo el mismo fruto a diferentes densidades relativas en condiciones de semicautiverio, puso en evidencia la preferencia $M$. nigra, incluso ante una abundancia elevada de $P$. carthagenensis y $R$. boliviensis. Este último fue, mas bien, el fruto más 
rechazado por $M$. gouazoubira. Dicha selección ocurre independientemente de la densidad de los frutos.

El comportamiento descrito de selección independiente de la densidad se puede explicar por tres razones: (1) La relación pulpa / semilla y la composición fitoquímica de $M$. nigra son óptimas para la dieta de la corzuela parda, (2) $M$. nigra es más palatable que $P$. carthagenensis y (3) El alto contenido de fibra de $R$. boliviensis hace que sea difícil de digerir, y por ende, es rechazado mayormente por $M$. gouazoubira.

\section{Agradecimientos}

Al personal técnico y científico de la Reserva Experimental Horco Molle por el apoyo en campo. Esta investigación fue realizada en el marco del Proyecto en Desarrollo - CIUNT (Expte 1173-97, Res. Rect. 0216 998) con el subsidio Nº.26 G130.

\section{Literatura citada}

Arias M.E. \& Parrado M.F. 1996. Estudio morfoanatómico de hoja y fruto de Psychotria carthagenensis Jacq. (Rubiaceae). Parodiana. 9 (1-2): 19-24.

Bell R.H.V. 1971. A grazing ecosystem in the Serengeti. Sci. Am. 225: 86-93.

Bodmer R.E. 1989. Frugivory in Amazonian Artiodactyla: evidence for the evolution of the ruminant stomach. J. Zool. Lond. 219: 457-467.

Bodmer R.E. 1991. Strategies of seed dispersal and seed predation in Amazonian ungulates. Biotropica. 23: 255261.

Branan W.V., Wekhoven M.C. \& Marchinton R.L. 1985. Food habits of brocket and white-tailed deer in Suriname. J. Wildl. Manage. 49: 972-976.

Braza F., Soriguer R.C., San José C., Delibes J.R., Aragón S., Fandos P. \& León L. 1994a. Métodos para el estudio y manejo de cérvidos. Ed. Junta de Andalucía. Consejería de Agricultura y Pesca. Dirección General de Desarrollo Forestal. Serie Monografía. Sevilla, España.

Braza F., San José C., Aragón S. \& Delibes J.R. 1994b. El corzo Andaluz. Ed. Junta de Andalucía. Consejería de Agricultura y Pesca. Dirección General de Desarrollo Forestal. Serie Monografía. Sevilla, España.

Cabrera A.L. 1971. Fitogeografía de la República Argentina. Bol. de la Sociedad Argentina de Botánica. 14: 1-42.

Capllonch P., Arias M.E. \& Parrado M.F. 2001. Interacciones entre Psychotria carthagenensis y la comunidad de frugívoros de Horco Molle, Tucumán. 9 pp.

Gwynne M.D. \& Bell R.H. 1968. Selection of vegetation components by grazing ungulates in the Serengeti National Park. Nature. 220: 390-393.

Hobbs N.T., Baker D.L. \& Gill R.B. 1983. Comparative nutritional ecology of montane ungulates during winter. $\mathrm{J}$ Wild Manage. 47: 1-16.

Juliá J.P., Richard E. \& Aceñolaza P. 1993. Observaciones sobre la alimentación del Wasú Virá (Mazama gouazoubira) en la Reserva Experimental de Horco
Molle (Universidad Nacional de Tucumán) (Tucumán Argentina). Yungas. 3: 4.

Mcarthur R.H. \& Pianka E.R. 1966. On the optimal use of patchy habitat. Am. Nat. 100: 601-609.

Nagy J.G. \& Haufler J.B. 1987. Nutrición de los animales silvestres. En: Manual de técnicas de gestión de vida silvestre, R.R. Tarrés (ed.), WWF.: 135-150.

Nudds T.D. 1980. Forage "preference": Theoretical considerations of diet selection by deer. J. Wildl. Manage. 44: 735-740.

Prado D.E. 1995. Selva Pedemontana: contexto regional y lista florística de un ecosistema en peligro. En: Brown, A. D. \& H. R. Grau (eds) Investigación, Conservación y desarrollo en Selvas subtropicales de montaña, Proyecto de Desarrollo Agroforestal - LIEY - UNT.: 19-52 Pulliam H.R. 1974. On the theory of optimal diets. Am. Nat. 108: 59-74.

Putman R. 1988. The natural history of deer. Comstock Publishing Associates, Cornell University Press. Ithaca, New York.

Richard E., Juliá J.P. \& Aceñolaza P.G. 1995a. Hábitos frugívoros de la corzuela parda (Mazama gouazoubira, Fischer, 1814) (Mammalia-Cervidae) en un ambiente secundario de Yungas. Doñana, Acta Vertebrata. 22 (12): 19-28.

Richard E., Juliá J.P., Samaniego E.J. \& Aceñolaza P. 1995b. La Corzuela Parda. Serie Monográfica y Didáctica 22. Fac. de Cs. Nat. e Inst. M. Lillo, UNT.: 35 Stallings J.R. 1984. Notes on feeding habits of Mazama gouazoubira in the Chaco Boreal of Paraguay. Biotropica. 16: 155-157.

Terborgh J. 1992. Maintenance of diversity in tropical forest. Biotropica. 24 (2b): 283-292.

Varela O. \& Brown A.D. 1995. Tapires y pecaríes como dispersores de plantas de los bosques húmedos subtropicales de Argentina. En: Investigación, Conservación y Desarrollo en Selvas Subtropicales de Montaña, Brown, A.D. y H.R. Grau (eds.). Proyecto se Desarrollo Agroforestal/LIEY.: 129-140.

Versvoorst F. 1982. Noroeste. P. 9-23. En Conservación de la vegetación natural de la República Argentina. FML, Ser. Conserv. Nat., 2: 127 p. Tucumán, Argentina.

Vides Almonacid R., Blendiger P., Rouges M. \& Alvarez M.E. 1993. El moradillo (Psychotria carthagenensis ",'que significa este nombre? Porque en cursiva??, es la familia de la planta?, es la subespecie?) como recurso para frugívoros en la estación seca de las selvas de montaña en Tucumán. Resumen de las Primeras Jornadas de Comunicaciones Internas de la Facultad de Ciencias Naturales e Instituto Miguel Lillo, Universidad Nacional de Tucumán.

Vides Almonacid R., Ayarde H., Scrocchi G., Romero F., Boero C. \& Chani J.M. 1998. Biodiversidad de Tucumán y el Noroeste Argentino. Opera Lilloana. 43. 89 pp.

Wilson M.F. \& Whelan C.J. 1993. Variation of dispersal phenology in a bird-dispersed shrub, Cornus drummondii. Ecol. Monog. 63: 151-17. 
Institución donde se realizó el trabajo:

1,2,3 LACEVEN (Laboratorio de Campo para el Estudio de Vertebrados Neotropicales), Facultad de Ciencias

Naturales e Instituto Miguel Lillo, P.O. Box \# 454; 4000 - Tucumán, ARGENTINA.

Institución actual:

${ }^{1}$ EcoDreams, Consultora Ecobiológica y Académica Internacional. División de Investigación. P.O. Box \# 180, La Paz, BOLIVIA. E-mail: chelonos@gmail.com

${ }^{2}$ Director del Instituto de Investigaciones y Desarrollo y Profesor Titular de Ecología Universidad de Aquino de Bolivia, La Paz, BOLIVIA.

${ }^{3}$ EcoDreams, Consultora Ecobiológica y Académica Internacional. División de Investigación. P.O. Box \# 180, La

Paz, BOLIVIA. E-mail: fonturbel@gmail.com

Dirección de contacto del autor para correspondencia:

* Francisco Fontúrbel R.

Casilla postal \# 180, La Paz, BOLIVIA

Tel./Fax +591-2-2423238; +591-72002766

Correo electrónico: fonturbel@gmail.com; fonturbel@yahoo.es 